\title{
Prescription Pattern of Chinese Herbal Products for Diabetes Mellitus in Taiwan: A Population-Based Study
}

\author{
Chung-Yu Huang, ${ }^{1,2}$ Yueh-Ting Tsai, ${ }^{1}$ Jung-Nien Lai, ${ }^{1,3}$ and Feng-Lin Hsu ${ }^{4}$ \\ ${ }^{1}$ Institute of Traditional Medicine, School of Medicine, National Yang-Ming University, No. 155, Section 2, Linong Road, \\ Taipei 112, Taiwan \\ ${ }^{2}$ Department of Traditional Medicine, Wan Fang Hospital, Taipei Medical University, Taipei 11696, Taiwan \\ ${ }^{3}$ Department of Chinese Medicine, Taipei City Hospital, Yangming Branch, Taipei 111, Taiwan \\ ${ }^{4}$ Graduate Institute of Pharmacognosy, College of Pharmacy, Taipei Medical University, No. 250, Wuxing Street, Taipei 11031, Taiwan
}

Correspondence should be addressed to Jung-Nien Lai; kareny@ms10.hinet.net

Received 23 December 2012; Revised 2 April 2013; Accepted 17 April 2013

Academic Editor: Srinivas Nammi

Copyright (C) 2013 Chung-Yu Huang et al. This is an open access article distributed under the Creative Commons Attribution License, which permits unrestricted use, distribution, and reproduction in any medium, provided the original work is properly cited.

\begin{abstract}
Background. Traditional Chinese medicine (TCM), when given as a therapy for symptom relief, has gained widespread popularity among diabetic patients. The aim of this study is to analyze the utilization of TCM among type 2 diabetic patients in Taiwan. Methods. The use of TCM for type 2 diabetic patients were evaluated using a randomly sampled cohort of 1,000,000 beneficiaries recruited from the National Health Insurance Research Database. Results. Overall, $77.9 \%(n=31,289)$ of type 2 diabetic patients utilized TCM and 13.9\% $(n=4,351)$ of them used TCM for the treatment of type 2 diabetes. Among the top ten most frequently prescribed herbal formulae, four remedies, Zhi-Bo-Di-Huang-Wan, Qi-Ju-Di-Huang-Wan, Ji-Sheng-Shen-Qi-Wan and Ba-Wei-DiHuang-Wan are derivative formulae of Liu-Wei-Di-Huang-Wan. In other words, Liu-Wei-Di-Huang-Wan and its derivatives were found to be the most common herbal formulae prescribed by TCM doctors for the treatment of diabetes in Taiwan. Conclusion. Although some evidence does support the use TCM to treat diabetes, the results from the current study may have been confounded by placebo effect, which emphasize the need for well conducted, double-blind, randomized, placebo-controlled studies in order to further evaluate the efficacy of Liu-Wei-Di-Huang-Wan on patients with type 2 diabetes.
\end{abstract}

\section{Introduction}

Type 2 diabetes is becoming a pandemic disorder, and the related alarming increase in the prevalence of both microvascular and macrovascular disease has raised significant concerns [1-7]. Importantly, clinically significant morbidity is present at diagnosis, but the development of diabetes-related microvascular and macrovascular diseases may occur much earlier and well before diagnosis. Due to a lack of appropriate care for preclinical diabetes, diabetes expenditures have grown dramatically annually due to increased medical care required by patients with diabetes-related complications. Even worse, although many drugs improve glycemic control, they do not necessarily provide real-world benefits. Previous reports have indicated that combination therapy with metformin and glyburide and the use of thiazolidinediones increase the risk of a composite end point involving cardiovascular events and mortality $[8,9]$. In addition, some diabetes medication unfortunately results in a number of common side effects such as nausea or upset stomach; these unwanted conditions drive patients to seek alternative advice [10]. Therefore, despite recent advances in intensive glycemic control, diabetes mellitus continues to be an important public health concern because it causes substantial morbidity and mortality as well as long-term complications [11, 12]. Not surprisingly, alternative therapies have become increasingly popular and are quickly approaching conventional therapy in their frequency of use as a treatment for diabetes and/or diabetes-related complications [13-15].

Previous studies of traditional Chinese medicines have found that tianhuafen (Radix Trichosanthis) [16], gegen (Radix Puerariae) [17], maimendong (Radix Ophiopogonis) [18], 
rougui (Cortex Cinnamomi) [19, 20], huangbai (Cortex Phellodendri) [21] and aconitum (Aconitum carmichaeli) [22] may have antidiabetic activity, and dihuang (Radix Rehmanniae) [23], shanzhuyu (Fructus Corni) [24], renshen (Radix Ginseng) [25], gancao (Radix Glycyrrhizae Praeparata) [26], and bitter orange (Aurantii Fructus) [27] have been suggested to increase insulin secretion. Unfortunately, evidence obtained in human studies is limited regarding patterns of use of classical traditional Chinese medicine (TCM) in relation to type 2 diabetes, which seem to be an area in which complementary and alternative medicines have recently grown in popularity. Furthermore, TCMs now seems to be marketed without established efficacy or safety in many Western countries $[28,29]$. Owing to the previously and to a lack of knowledge about the prescription profile of TCMs, researchers and conventional doctors have found it difficult to explore the potential mechanisms of TCM therapies targeting type 2 diabetes. Furthermore, it also has proved difficult to assess the cost effectiveness of using TCM therapy and to observe the interaction between Chinese herbs and conventional diabetes drugs.

TCM, which includes acupuncture, traumatology manipulative therapies, and Chinese herbal products, has been an important part of health care in Taiwan for hundreds of years and is fully reimbursed under the current National Health Insurance (NHI) system. Previous studies using the NHI research database have reported that female [30] and individuals aged in their 30s [31] are more likely to use TCM among the general population in Taiwan. Chinese herbal remedies are the most common TCM modality used by this population, followed by acupuncture and traumatology manipulative therapies. The unique approach to TCM diagnosis involves gathering clinical symptoms and signs, and then a treatment principle is put forward in accordance with the aforementioned diagnostic process. In this situation, researchers in Taiwan have found that symptoms, signs, and ill-defined conditions are one of the most common reasons for TCM visits across various different patient populations [32-34]. Accordingly, the claims that database, part of the Taiwan National Health Insurance Research Database, provides are a platform for understanding the utilization of TCM therapies by licensed TCM doctors $[30,31,35]$. The aim of our study is to analyze a random sample from this comprehensive database and to determine the TCM utilization patterns of newly diagnosed type 2 diabetes patients in Taiwan. The results of this study should provide valuable information that will enable physicians to respond to patient use of TCM in an informed way, which will in turn strengthen further the patient-physician relationship when treating diabetes and diabetes-related complications.

\section{Materials and Methods}

2.1. Data Resources. This study was designed as a populationbased study analyzing a sample of one million subjects selected at random from the 22 million beneficiaries of the National Health Insurance scheme of Taiwan and aimed to determine the prevalence of using prescribed Chinese herbal prescriptions (CHP) among diabetes patients between January 1 ,

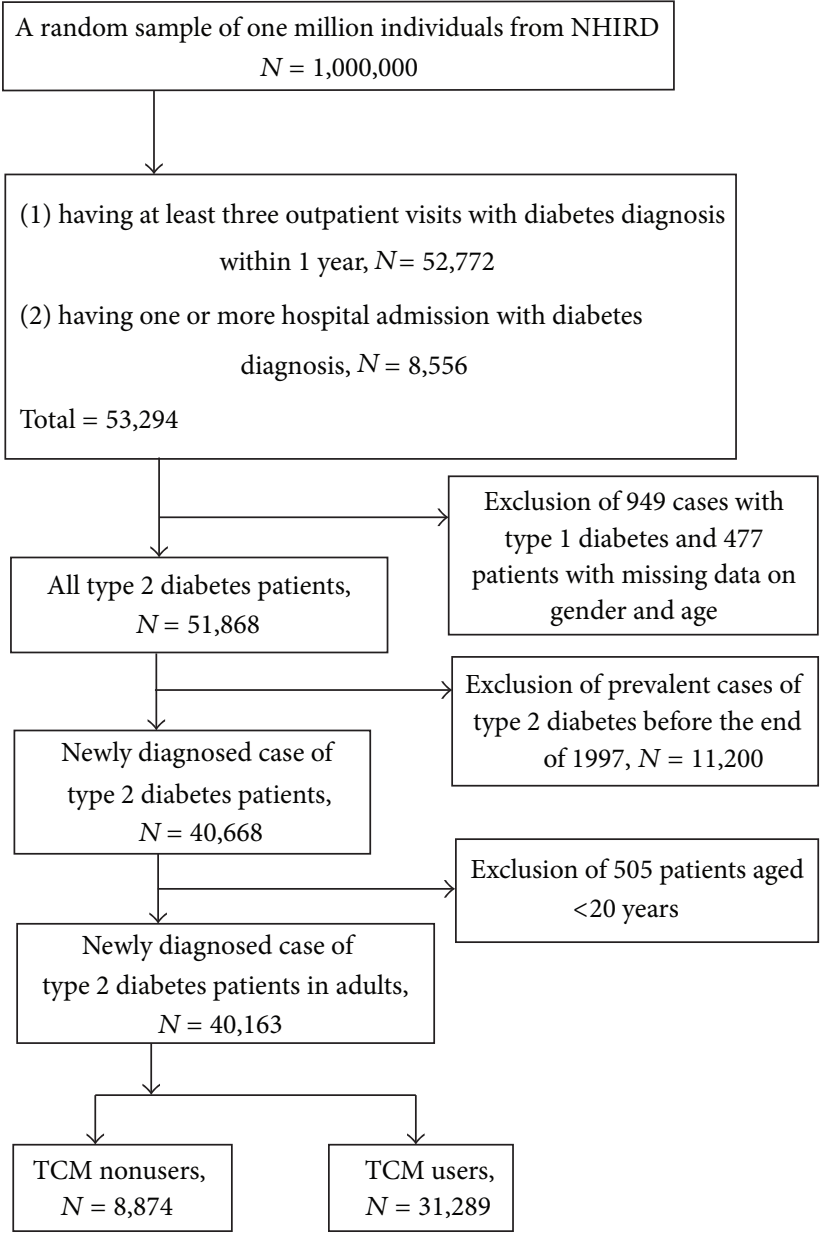

Figure 1: Flow recruitment chart of subjects from the one million random samples obtained from the National Health Insurance Research Database (NHIRD), 1997 to 2008, in Taiwan.

1998 and December 31, 2008. All data were obtained from the National Health Insurance Research Database (NHIRD), which includes all the reimbursement data of the NHI with the identification numbers of all individuals being encrypted and transformed; this database is maintained by the National Health Research Institutes of Taiwan [36]. The NHIRD database contains patient's gender and date of birth, all records of clinical visits and hospitalization, prescribed drugs and dosages, including CHP, and three major diagnoses coded in the International Classification of Diseases, Ninth Revision, and Clinical Modification (ICD-9-CM) formats [37].

2.2. Study Subjects. The selection of study subjects from the random sample of one million individuals was performed as follows (Figure 1). First, we included all patients that (1) had at least three outpatient visits with a diabetes diagnosis within 1 year $(n=52,772)$ or $(2)$ having one or more hospital admission with diabetes diagnosis $(n=8,556)$ [38]. A total of 53,294 subjects were obtained. Second, we excluded all patients with type 1 diabetes $(n=949)$ or with missing information on gender and age $(n=477)$. Third, cases of diabetes 
$(n=11,200)$ that had been diagnosed before the end of 1997 were also excluded to ensure that all the subjects included were newly diagnosed with type 2 diabetes in the time period 1998-2008. Fourth, subjects under 20 years of age $(n=505)$ were also excluded to limit the study sample to adults. Finally, 40,163 study subjects were included in the study cohort.

2.3. Study Variables. To determine the key independent variables for utilization of TCM among diabetes patients, we selected a series of demographic factors based on previous studies [3, 38-41]. The subjects were categorized into four groups according to age: $20-39,40-59,60-79$, and $\geq 80$ years. Taiwan was divided into seven geographic regions: Taipei city, Kaohsiung city, Northern region, Central region, Southern region, Eastern region and Outlying islands. We split the monthly wage of individuals into four levels: new Taiwan dollars (NT\$) $0,1-19,999,20,000-39,999$, and $\geq 40,000$. We also searched the NHIRD database for clinical complications and treatment records related to diabetes as independent variables. The complications associated with diabetes included nephropathy (ICD-9 code, 581.81, 583.81, 585.1-585.9), retinopathy (362.01-362.07, 365.44, 366.41, 369.00-369.9), neuropathy $(353.5,536.3,354.0-355.9,713.5,337.1,357.2)$, peripheral circulatory disorders (250.70), and other specified manifestations (250.80) [42]. The reimbursement database contains all details related to the prescription of conventional medicines for treating diabetes mellitus. Then, for the final analysis, we categorized the types of preparations into the following categories: sulfonylureas, biguanides, $\alpha$-glucosidase, meglitinide, thiazolidinediones, guar gum, and insulin.

2.4. Statistical Analysis. Data analysis consisted of descriptive statistics, including the prescription rates of TCM users stratified by patient's demographic characteristics, indications for the prescription of TCM, and the most frequently prescribed herbal formulae used when treating diabetes. Primary indications were classified according to their ICD-9 code. The diagnoses were coded according to the ICD-9 and grouped into a series of distinct broad disease categories. The potential effects of Chinese herbs contained in the ten most commonly prescribed CHPs were grouped according to previous in vivo and in vitro studies and are summarized in Table 4 [16-27]. Multiple logistic regression was conducted to evaluate the factors that correlated with TCM use. A significance level of $\alpha=0.05$ was selected. The statistical software package SAS 9.13 was used for data management and analysis.

\section{Results}

The database of outpatient claims contained information on 40,163 patients with type 2 diabetes from 1998 to 2008 . Among them, 31,289 (77.9\%) patients used TCM outpatient services at least once. Most TCM users (91.2\%) also received diabetes treatment. Among all TCM users, $13.9 \%(n=4,351)$ used TCM for the treatment of type 2 diabetes. Details of the demographic distribution of TCM users and nonusers are presented in Table 1. The mean age of TCM nonusers was slightly higher than that of TCM users. There were more
TCM users than TCM nonusers with an income level of NT\$ 20,000-39,999 or residing in Central Taiwan.

The adjusted odds ratios (aORs) and 95\% confidence intervals (95\% CIs) obtained by multiple logistic regression are also presented in Table 1. Compared with the age group $40-59$ years $(\mathrm{aOR}=1.00)$, those aged $20-39$ years were more likely to be TCM users. There was also a significant difference between TCM users and nonusers with there being more of the former in the income group of NT\$20,000-39,999. After adjusting for other factors, patients with more type 2 diabetes chronic complications (one complication: OR $=1.10,95 \%$ CI: $1.04-1.16$; two complications: OR $=1.28$, 95\% CI: $1.19-$ 1.38; more than three complications: $\mathrm{OR}=1.25$, 95\% CI: $1.13-$ 1.38) were more likely to seek TCM treatment than those with no chronic complication $(\mathrm{aOR}=1.00)$. There was no significant difference in the diabetes treatment modalities received (monotherapy ( $\mathrm{aOR}=1.00$ ) or combination therapy) between TCM users and TCM nonusers, except among those who took more than five types of antidiabetic drugs (OR $=1.13$, 95\% CI: 1.01-1.26) for the control of blood sugar or HbAlc.

Among the diabetes patients visiting TCM doctors, 3,627,622 (92.6\%) visits involved the prescription of TCM, while the rest were prescribed acupuncture and traumatology manipulative therapies. Analysis of the major disease categories for all TCM visits made by 31,289 TCM users are summarized in Table 2. The findings show that "symptoms, signs, and ill-defined conditions" were the most common reason for using Chinese herbal prescriptions (CHP) (16.8\%, $n=608,535)$, followed by "endocrine, nutritional and metabolic diseases, and immunity disorders" (12.1\%, $n$ $=439,612)$, and "diseases of digestive system" $(11.5 \%, n=$ 417,611). Details of the most frequently prescribed CHP for treating type 2 diabetes by TCM doctors are provided in Table 3. Liu-Wei-Di-Huang-Wan (Rehmannia six pill) was the most frequently prescribed CHP, followed by $\mathrm{Bai}-\mathrm{Hu}$ Jia-Ren-Shen-Tang (white tiger plus ginseng combination), Zhi-Bo-Di-Huang-Wan (zhibai Rehmannia six pill), Qi-Ju-DiHuang-Wan (chichu Rehmannia pill), Yu-Quan-Wan (jade spring pill), Ji-Sheng-Shen-Qi-Wan (economic health shenqi pill), Xue-Fu-Zhu-Yu-Tang (persica and achyranthes combination), Ba-Wei-Di-Huang-Wan (eight-flavour Rehmannia pill), Bai-Hu-Tang (white tiger combination), and Gan-LuYin (sweet combination drink). Among the top ten most frequently prescribed herbal formulae, Zhi-Bo-Di-HuangWan, Qi-Ju-Di-Huang-Wan, Ji-Sheng-Shen-Qi-Wan, and BaWei-Di-Huang-Wan are four derivative formulae of Liu-WeiDi-Huang-Wan, which all contain Rhizoma Rehmanniae Preparata, Fructus Corni, Rhizoma Dioscoreae, Rhizoma Alismatis, Cortex Moutan Radicis, and Poria. In other words, LiuWei-Di-Huang-Wan and its various derivatives are the most common herbal formulae prescribed by TCM doctors for the treatment of diabetes in Taiwan. The ten most frequently prescribed CHPs include Chinese herbs that have been historically used to lower serum glucose. The potential effects of these Chinese herbs when used to treat type 2 diabetes are summarized in Table 4 and include increasing insulin secretion, enhancing glucose uptake by adipose and muscle 
TABLE 1: Demographic characteristics and results of multiple logistic regressions showing the adjusted odds ratio (aOR) and 95\% CI (confidence interval) for diabetes from 1998 to 2008 in Taiwan.

\begin{tabular}{|c|c|c|c|c|}
\hline Characteristic & All patients & $\mathrm{TCM}^{\mathrm{a}}$ nonusers $(\%)$ & TCM users (\%) & $\mathrm{aOR}^{\mathrm{b}}\left(95 \% \mathrm{CI}^{\mathrm{c}}\right)$ \\
\hline Number of cases & 40,163 & 8,874 & 31,289 & \\
\hline \multicolumn{5}{|l|}{ Gender } \\
\hline Male & $20,971(52.2)$ & $5,718(64.4)$ & $15,253(48.7)$ & 1.00 \\
\hline Female & $19,192(47.8)$ & $3,156(35.6)$ & $16,036(51.3)$ & $1.98(1.88-2.08)$ \\
\hline \multicolumn{5}{|l|}{ Age at diagnosis (years) } \\
\hline Mean \pm SD & $56.7 \pm 12.4$ & $58.3 \pm 12.9$ & $56.3 \pm 12.2$ & \\
\hline $20 \sim 39$ & $3,175(7.9)$ & $609(6.9)$ & $2,566(8.2)$ & $1.11(1.01-1.22)$ \\
\hline $40 \sim 59$ & $20,523(51.1)$ & $4,184(47.1)$ & $16,339(52.2)$ & 1.00 \\
\hline $60 \sim 79$ & $15,244(38.0)$ & $3,653(41.2)$ & $11,591(37.0)$ & $0.79(0.75-0.83)$ \\
\hline$\geq 80$ & $1,221(3.0)$ & $428(4.8)$ & $793(2.5)$ & $0.46(0.41-0.52)$ \\
\hline \multicolumn{5}{|l|}{ Insured salaries (NT\$d/month) } \\
\hline 0 & $9,981(24.9)$ & $2,207(24.9)$ & $7,774(24.9)$ & 1.00 \\
\hline $1-19,999$ & $21,256(52.9)$ & $4,801(54.1)$ & $16,455(52.6)$ & $1.01(0.95-1.08)$ \\
\hline $20,000-39,999$ & $5,414(13.5)$ & $982(11.1)$ & $4,432(14.2)$ & $1.33(1.21-1.46)$ \\
\hline$\geq 40,000$ & $3,512(8.7)$ & $884(9.9)$ & $2,628(8.4)$ & $1.02(0.92-1.12)$ \\
\hline \multicolumn{5}{|l|}{ Insured region } \\
\hline Taipei city & $6,975(17.4)$ & $1,746(19.7)$ & $5,229(16.7)$ & 1.00 \\
\hline Kaohsiung city & $2,802(7.0)$ & $606(6.8)$ & $2,196(7.0)$ & $1.21(1.09-1.35)$ \\
\hline Northern Taiwan & $11,316(28.2)$ & $2,652(29.9)$ & $8,664(27.7)$ & $1.10(1.02-1.18)$ \\
\hline Central Taiwan & $7,172(17.8)$ & $1,070(12.1)$ & $6,102(19.5)$ & $1.92(1.76-2.09)$ \\
\hline Southern Taiwan & $10,447(26.0)$ & $2,436(27.4)$ & $8,011(25.6)$ & $1.12(1.04-1.20)$ \\
\hline Eastern Taiwan & $1,130(2.8)$ & $288(3.3)$ & $842(2.7)$ & $0.97(0.83-1.12)$ \\
\hline Outlying islands & $319(0.8)$ & $75(0.8)$ & $244(0.8)$ & $1.11(0.85-1.45)$ \\
\hline \multicolumn{5}{|l|}{ Number of diabetic complications } \\
\hline 0 & $17,913(44.6)$ & $4,213(47.5)$ & $13,700(43.8)$ & 1.00 \\
\hline 1 & $12,833(32.0)$ & $2,823(31.8)$ & $10,010(32.0)$ & $1.10(1.04-1.16)$ \\
\hline Nephropathy & $2,990(7.4)$ & $724(8.1)$ & $2,266(7.2)$ & \\
\hline Retinopathy & $2,764(6.9)$ & $674(7.6)$ & $2,090(6.7)$ & \\
\hline Neuropathy & $5,339(13.3)$ & $993(11.2)$ & $4,346(13.9)$ & \\
\hline Peripheral circulatory disorders (PCD) & $844(2.1)$ & $213(2.4)$ & $631(2.0)$ & \\
\hline Other specified manifestations & $896(2.2)$ & $219(2.5)$ & $677(2.2)$ & \\
\hline 2 & $6,296(15.7)$ & $1,226(13.8)$ & $5,070(16.2)$ & $1.28(1.19-1.38)$ \\
\hline Nephropathy + retinopathy & $1,039(2.6)$ & $241(2.7)$ & $798(2.5)$ & \\
\hline Nephropathy + neuropathy & $1,588(4.0)$ & $300(3.4)$ & $1,288(4.1)$ & \\
\hline Nephropathy + PCD & $256(0.6)$ & $64(0.7)$ & $192(0.6)$ & \\
\hline Nephropathy + others & $273(0.7)$ & $61(0.7)$ & $212(0.7)$ & \\
\hline Retinopathy + neuropathy & $1,579(3.9)$ & $250(2.8)$ & $1,329(4.3)$ & \\
\hline Retinopathy + PCD & $234(0.6)$ & $45(0.5)$ & $189(0.6)$ & \\
\hline Retinopathy + others & $206(0.5)$ & $46(0.5)$ & $160(0.5)$ & \\
\hline Neuropathy + PCD & $582(1.4)$ & $107(1.2)$ & $475(1.5)$ & \\
\hline Neuropathy $1+$ others & $429(1.1)$ & $81(0.9)$ & $348(1.1)$ & \\
\hline PCD + others & $110(0.3)$ & $31(0.4)$ & $79(0.3)$ & \\
\hline$\geq 3$ & $3,121(7.7)$ & $612(6.9)$ & $2,509(8.0)$ & $1.25(1.13-1.38)$ \\
\hline
\end{tabular}


TABle 1: Continued.

\begin{tabular}{|c|c|c|c|c|}
\hline Characteristic & All patients & $\mathrm{TCM}^{\mathrm{a}}$ nonusers $(\%)$ & TCM users (\%) & $\mathrm{aOR}^{\mathrm{b}}\left(95 \% \mathrm{CI}^{\mathrm{c}}\right)$ \\
\hline \multicolumn{5}{|c|}{ Number of medical treatments for diabetes } \\
\hline None & $3,370(8.4)$ & $627(7.1)$ & $2,743(8.8)$ & \\
\hline Monotherapy $^{e}$ & $6,048(15.1)$ & $1,443(16.3)$ & $4,605(14.7)$ & 1.00 \\
\hline Two-drug combination ${ }^{\mathrm{f}}$ & $13,609(33.9)$ & $3,127(35.2)$ & $10,482(33.5)$ & $1.01(0.94-1.09)$ \\
\hline Three-drug combination & $8,675(21.6)$ & $1,937(21.8)$ & $6,738(21.5)$ & $1.00(0.93-1.09)$ \\
\hline Four-drug combination & $5,026(12.5)$ & $1,093(12.3)$ & $3,933(12.6)$ & $1.01(0.92-1.10)$ \\
\hline Over five-drug combination & $3,435(8.5)$ & $647(7.3)$ & $2,788(8.9)$ & $1.13(1.01-1.26)$ \\
\hline
\end{tabular}

${ }^{\mathrm{a}} \mathrm{TCM}$ refers to traditional Chinese medicine; ${ }^{\mathrm{b}}$ OR refer to odds ratio; ${ }^{\mathrm{c}} \mathrm{CI}$ refers to confidence interval; ${ }^{\mathrm{d}} \mathrm{NT} \$$ refers to new Taiwan dollars, of which US\$ $1=$ NT\$30 approximately.

${ }^{\mathrm{e}}$ Monotherapy is the use of a single antidiabetic drug (sulfonylureas, biguanides, $\alpha$-glucosidase, meglitinide, thiazolidinediones, guar gum, or insulin).

$\mathrm{f}$ combination therapy is the use of more than one antidiabetic drug.

tissues, inhibiting glucose absorption from intestine, inhibiting glucose production from hepatocytes, and decreasing insulin resistance or enhancing insulin sensitivity.

\section{Discussion}

The prevalence of type 2 diabetes in Taiwan over the 11 years in the study was $4.0 \%$, which is in line with the estimates given by previous surveys $[1,43]$. Worthy of note, the utilization of TCM among adults with type 2 diabetes in Taiwan during the study period was $77.9 \%$, which appears to be high compared with previous findings $[14,15]$. TCM is a unique traditional therapy approach for various ailments that has been used in Taiwan for over hundreds of years, and this long period of use may contribute significantly to the high prevalence of TCM usage among type 2 diabetic subjects. In addition, it should be noted that TCM treatment is covered by the NHI system. Therefore, unsurprisingly, the prevalence of CHP for treating type 2 diabetes among adults is comparatively higher in Taiwan than in other countries $[15,44]$. The present study includes all patients who were newly diagnosed with type 2 diabetes by qualified conventional doctors between 1998 and 2008 from a random sample of one million subjects among the insured general population; importantly the rate of insured individuals has been consistently above $96 \%$ since 1997 , and therefore we can rule out the possibility of selection bias.

The present results show that, although $91 \%$ of type 2 diabetic patients in Taiwan have received antidiabetic treatment, over half of them still have suffered from one or more diabetes complications during the 11-year follow-up. Nephropathy and neuropathy were the two most common diabetes complications. One possibility is that type 2 diabetes has a long asymptomatic preclinical phase that is likely to go undetected [45-49], and the injurious effects of asymptomatic hyperglycemia, therefore, have resulted in a high incidence of microvascular and macrovascular complications [4, 42]. The present study found that patients with type 2 diabetes who developed more than one site of involvement re-diabetes complications were more likely to seek advice from a TCM doctor. However, regardless of their experience in receiving more than one type of antidiabetic drug with the aim of improving their poor control of serum blood sugar, the choice of any of the major medical options available to patients with type 2 diabetes was not associated with the use of TCM. Hence, we suggest that when TCM is used to treat type 2 diabetes in Taiwan, this is generally an adjunct to diabetes treatment rather than a replacement for it.

The present findings show that, among diabetes patients, females and those aged 20-39 years were more likely to be TCM users than males and other age groups as shown in Table 1. As shown in Table 2, "symptoms, signs, and ill-defined conditions" were the most common reasons for using CHP $(16.8 \%, n=608,535)$, followed by "endocrine, nutritional and metabolic diseases, and immunity disorders" $(12.1 \%, n$ $=439,612)$ and "diseases of digestive system" $(11.5 \%, n=$ $417,611)$. Further analysis found that TCM doctors tended to use Chinese herbal remedies targeting diabetes as well as gastrointestinal disorders that might be the uncomfortable side effects of diabetes drugs. Although previous studies have demonstrated that acupuncture might be related to an alternative therapy for treating hyperglycemia and diabetes complications, the present study indicated that acupuncture in Taiwan is used by this study population mainly for diseases of the musculoskeletal system and connective tissue.

Liu-Wei-Di-Huang-Wan was the most frequently prescribed formula for treating type 2 diabetes in Taiwan during the study period, as shown in Table 3. Liu-Wei-Di-HuangWan is among the most highly regarded ancient Chinese herbal formulae and was first documented in the classical Chinese text Xiao Er Yao Zheng Zhi Jue (Key to Therapeutics of Children's Diseases) circa 1119 A.D. In the classical literature, Liu-Wei-Di-Huang-Wan is said to nourish yin and to invigorate the kidney, which might indicate it as a potentially efficacious therapy for reducing hyperglycemia and relieving neuropathic and nephropathic complications in diabetes mellitus [50-53]. Among the top ten most frequently prescribed formulae for treating type 2 diabetes, $Z$ hi-Bo-DiHuang-Wan, Qi-Ju-Di-Huang-Wan, Ji-Sheng-Shen-Qi-Wan, and Ba-Wei-Di-Huang-Wan, which are all derivatives of LiuWei-Di-Huang-Wan, are prescribed to alleviate various common symptoms of type 2 diabetes, namely, unusual thirst, blurred vision, frequent urination, and cold feeling in the 
TABLE 2: Frequency distribution of traditional Chinese medicine (TCM) visits by major disease categories (according to 9th ICD codes) among diabetes patients from 1998 to 2008 in Taiwan.

\begin{tabular}{|c|c|c|c|c|}
\hline \multirow[b]{2}{*}{ Major disease category } & \multirow[b]{2}{*}{ ICD-9-CM codes } & \multicolumn{3}{|c|}{ No. of visits (no. of patients) } \\
\hline & & Chinese herbal remedies & $\begin{array}{c}\text { Acupuncture or } \\
\text { manipulative therapies }\end{array}$ & Total of TCM \\
\hline Infectious and parasitic diseases & 001-139 & $21,083(761)$ & $69(13)$ & $21,152(772)$ \\
\hline Neoplasms & $140-239$ & $33,132(366)$ & $613(20)$ & $33,745(381)$ \\
\hline $\begin{array}{l}\text { Endocrine, nutritional and } \\
\text { metabolic diseases, and } \\
\text { immunity disorders }\end{array}$ & $240-279$ & $439,612(5,565)$ & $1,995(127)$ & $441,607(5,620)$ \\
\hline Diabetes & 250 & $377,621(4,328)$ & $1,505(65)$ & $379,126(4,350)$ \\
\hline Others & & $61,991(1,703)$ & $490(62)$ & $62,481(1,742)$ \\
\hline Mental disorders & $290-319$ & $24,834(858)$ & $536(28)$ & $25,370(876)$ \\
\hline $\begin{array}{l}\text { Diseases of nervous system and } \\
\text { sense organs }\end{array}$ & $320-389$ & $104,033(3,962)$ & $4,593(802)$ & $108,626(4,513)$ \\
\hline Diseases of circulatory system & $390-459$ & $180,821(3,647)$ & $5,857(444)$ & $186,678(3,892)$ \\
\hline Diseases of respiratory system & $460-519$ & $377,262(10,505)$ & $1,423(126)$ & $378,685(10,537)$ \\
\hline Diseases of digestive system & $520-579$ & $417,611(9,387)$ & $1,332(129)$ & $418,943(9,432)$ \\
\hline Diseases of genitourinary system & $580-629$ & $171,262(4,145)$ & $1,294(63)$ & $172,556(4,170)$ \\
\hline $\begin{array}{l}\text { Diseases of skin and } \\
\text { subcutaneous tissue }\end{array}$ & $680-709$ & $61,387(2,784)$ & $280(41)$ & $61,667(2,810)$ \\
\hline $\begin{array}{l}\text { Diseases of musculoskeletal } \\
\text { system and connective tissue }\end{array}$ & $710-739$ & $318,920(8,808)$ & $82,936(12,682)$ & $401,856(16,932)$ \\
\hline $\begin{array}{l}\text { Symptoms, signs, and ill-defined } \\
\text { conditions }\end{array}$ & $780-799$ & $608,535(14,216)$ & $3,839(458)$ & $612,374(14,345)$ \\
\hline Injury and poisoning & $800-999$ & $17,994(1,389)$ & $90,542(14,141)$ & $108,536(14,625)$ \\
\hline \multirow[t]{2}{*}{ Supplementary classification $^{+}$} & V01-V82, & $115(9)$ & $0(0)$ & $115(9)$ \\
\hline & E800-E999 & $0(0)$ & $0(0)$ & $0(0)$ \\
\hline Others $^{*}$ & & $851,021(15,034)$ & $96,311(11,164)$ & $947,332(18,954)$ \\
\hline Total & & $3,627,622(27,135)$ & $291,620(22,891)$ & $3,919,242(31,289)$ \\
\hline
\end{tabular}

TABLE 3: Ten most common herbal formulae prescribed by TCM doctors for the treatment of type 2 diabetes among 31,289 patients from 1998 to 2008 in Taiwan.

\begin{tabular}{|c|c|c|c|c|}
\hline Herbal formulae & English name & $\begin{array}{c}\text { Number of } \\
\text { person-days } \\
N=775,447(\%)\end{array}$ & Average daily dose (g) & $\begin{array}{l}\text { Average duration for } \\
\text { prescription (days) }\end{array}$ \\
\hline Liu-Wei-Di-Huang-Wan & Rehmannia six pill & $62,249(8.0)$ & 8.0 & 47.9 \\
\hline Bai-Hu-Jia-Ren-Shen-Tang & $\begin{array}{l}\text { White tiger plus ginseng } \\
\text { combination }\end{array}$ & $42,676(5.5)$ & 7.5 & 47.4 \\
\hline Zhi-Bo-Di-Huang-Wan & Zhibai Rehmannia six pill & $37,918(4.9)$ & 5.7 & 45.4 \\
\hline Qi-Ju-Di-Huang-Wan & Chichu Rehmannia pill & $37,796(4.9)$ & 5.7 & 64.4 \\
\hline Yu-Quan-Wan & Jade spring pill & $35,878(4.6)$ & 5.6 & 54.8 \\
\hline Ji-Sheng-Shen-Qi-Wan & $\begin{array}{l}\text { Economic health shenqi } \\
\text { pill, life-saving renal Chi } \\
\text { pill }\end{array}$ & $27,347(3.5)$ & 7.1 & 43.6 \\
\hline Xue-Fu-Zhu-Yu-Tang & $\begin{array}{l}\text { Persica and achyranthes } \\
\text { combination }\end{array}$ & $22,708(2.9)$ & 5.3 & 45.9 \\
\hline Ba-Wei-Di-Huang-Wan & $\begin{array}{l}\text { Eight-flavour Rehmannia } \\
\text { pill }\end{array}$ & $19,247(2.5)$ & 9.1 & 41.5 \\
\hline Bai-Hu-Tang & White tiger combination & $18,801(2.4)$ & 7.0 & 42.1 \\
\hline Gan-Lu-Yin & Sweet combination drink & $18,502(2.4)$ & 5.1 & 38.1 \\
\hline
\end{tabular}


TABLE 4: Potential effects of herbs present in the ten most common herbal formulae prescribed by TCM doctors for treating type 2 diabetes.

\begin{tabular}{|c|c|c|}
\hline Herbal formulae & Number of herbs & Ingredient herbs \\
\hline Liu-Wei-Di-Huang-Wan & 6 & 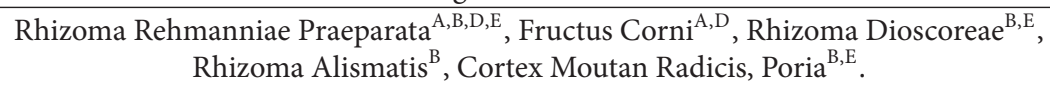 \\
\hline Bai-Hu-Jia-Ren-Shen-Tang & 5 & $\begin{array}{c}\text { Gypsum Fibrosum, Rhizoma Anemarrhenae }{ }^{\mathrm{E}} \text {, Radix Glycyrrhizae Praeparata }{ }^{\mathrm{A}} \text {, } \\
\text { Semen Oryzae Sativae, Radix Ginseng }{ }^{\mathrm{A}, \mathrm{B}, \mathrm{C}, \mathrm{D}} \text {. }\end{array}$ \\
\hline Zhi-Bo-Di-Huang-Wan & 8 & $\begin{array}{c}\text { Rhizoma Anemarrhenae }{ }^{\mathrm{E}} \text {, Cortex Phellodendri, Rhizoma Rehmanniae } \\
\text { Praeparata }^{\mathrm{A}, \mathrm{B}, \mathrm{D}, \mathrm{E}} \text {, Fructus Corni } \mathrm{F}^{\mathrm{A}, \mathrm{D}} \text {, Rhizoma Dioscoreae } \mathrm{e}^{\mathrm{B}, \mathrm{E}}, \text { Rhizoma Alismatis }^{\mathrm{B}} \text {, } \\
\text { Cortex Moutan Radicis, Poria }{ }^{\mathrm{B}, \mathrm{E}} \text {. }\end{array}$ \\
\hline Qi-Ju-Di-Huang-Wan & 8 & $\begin{array}{l}\text { Flos Chrysanthemi, Fructus Lycii }{ }^{\mathrm{B}, \mathrm{E}} \text {, Rhizoma Rehmanniae Praeparata }{ }^{\mathrm{A}, \mathrm{B}, \mathrm{D}, \mathrm{E}} \text {, } \\
\text { Fructus Corni }^{\mathrm{A}, \mathrm{D}} \text {, Rhizoma Dioscoreae, Rhizoma Alismatis }{ }^{\mathrm{B}} \text {, Cortex Moutan } \\
\text { Radicis, Poria }{ }^{\mathrm{B}, \mathrm{E}} \text {. }\end{array}$ \\
\hline Yu-Quan-Wan & 9 & $\begin{array}{c}\text { Radix Trichosanthis, Radix Puerariae }{ }^{\mathrm{A}, \mathrm{B}}, \text { Radix Ophiopogonis }^{\mathrm{A}, \mathrm{C}, \mathrm{D}}, \text { Radix Ginseng, } \\
\text { Poria }^{\mathrm{B}, \mathrm{E}}, \text { Radix Astragali }^{\mathrm{B}, \mathrm{E}} \text {, Radix Glycyrrhizae Praeparata, Fructus Mume, Radix } \\
\text { Astragali Praeparata. }\end{array}$ \\
\hline Ji-Sheng-Shen-Qi-Wan & 10 & $\begin{array}{l}\text { Semen Plantaginis, Radix Achyranthis Bidentatae, Ramulus Cinnamomi, Radix } \\
\text { Aconiti, Rhizoma Rehmanniae Praeparata, Fructus Corni, Rhizoma Dioscoreae }{ }^{\mathrm{B}, \mathrm{E}} \text {, } \\
\text { Rhizoma Alismatis }{ }^{\mathrm{B}} \text {, Cortex Moutan Radicis, Poria }{ }^{\mathrm{B}, \mathrm{E}} \text {. }\end{array}$ \\
\hline Xue-Fu-Zhu-Yu-Tang & 11 & $\begin{array}{c}\text { Chinese Angelia Root, Rhizoma Rehmanniae Praeparata, Peach Kernel, Safflower, } \\
\text { Bitter Orange }{ }^{\mathrm{A}} \text {, Red Peony Root, Bupleurum Root, Glycyrrhiza, Platycodon Root, } \\
\text { Chuanxiong Rhizome, Cyathula Root. }\end{array}$ \\
\hline Ba-Wei-Di-Huang-Wan & 8 & $\begin{array}{l}\text { Ramulus Cinnamomi }{ }^{\mathrm{E}} \text {, Radix Aconiti }{ }^{\mathrm{B}} \text {, Rhizoma Rehmanniae Praeparata, Fructus } \\
\text { Corni, Rhizoma Dioscoreae, Rhizoma Alismatis, Cortex Moutan Radicis, Poria }{ }^{\mathrm{B}, \mathrm{E}} \text {. }\end{array}$ \\
\hline Bai-Hu-Tang & 4 & $\begin{array}{c}\text { Gypsum Fibrosum, Rhizoma Anemarrhenae, Radix Glycyrrhizae Praeparata, } \\
\text { Semen Oryzae Sativae. }\end{array}$ \\
\hline Gan-Lu-Yin & 10 & $\begin{array}{c}\text { Rhizoma Rehmanniae, Radix Ophiopogonis }{ }^{\mathrm{A}, \mathrm{C}, \mathrm{D}} \text {, Radix Glycyrrhizae Praeparata, } \\
\text { Herba Dendrobii, Radix Asparagi, Eriobotryae Folium, Bitter Orange }{ }^{\mathrm{A}} \text {, Scutellariae } \\
\text { radix, Wormwood Herb, Rhizoma Rehmanniae Praeparata. }\end{array}$ \\
\hline
\end{tabular}

${ }^{\mathrm{A}}$ Increase in insulin secretion, ${ }^{\mathrm{B}}$ enhancement of glucose uptake by adipose and muscle tissues, ${ }^{\mathrm{C}}$ inhibition of glucose absorption by the intestine, ${ }^{\mathrm{D}}$ inhibition of glucose production by hepatocytes, and ${ }^{\mathrm{E}}$ decrease in insulin resistance or enhancement of insulin sensitivity.

limbs, respectively. Other frequently prescribed formulae are associated with severe dysphoric thirst and lassitude (BaiHu-Jia-Ren-Shen-Tang or white tiger plus ginseng decoction, $\mathrm{Bai}-\mathrm{Hu}$-Tang or white tiger decoction, Yu-Quan-Wan or jade spring combination, and Gan-Lu-Yin or sweet dew decoction or sweet combination drink) and with peripheral neuropathy due to blood stasis (Xue-Fu-Zhu-Yu-Tang or Persica and Carthamus Combination). Although, previous in vitro studies have found that some Chinese herbs are able to decrease serum levels of glucose, glycosylated proteins, and hemoglobin $\mathrm{AlC}$, possibly by blocking intestinal absorption and/or inhibiting hepatic glucose-6-phosphatase $[50,54]$, there have not yet been any clinical trials that have demonstrated the efficacy and safety of Liu-Wei-Di-Huang-Wan and its derivatives when treating diabetes type 2. In general, TCM doctors treated diabetes patients' complaints according to the syndrome differentiation theory rather than by making a specific diagnosis; this is based on holistic consideration of diabetes patients who are suffering from symptoms and complications at various sites. In this context and in line with previous results [51], the present study found that TCM doctors in Taiwan prescribed herbal therapies mainly to optimize the body's ability to function normally, rather than as a cure for diabetes. Moreover, despite inadequate data on the clinical safety and efficacy of CHP when treating diabetic patients, a large number of patients use them. Thus, based on the present trend in TCM utilization, herbal remedies for treating diabetes and/or diabetic complications will continue to be used. Although we respect the patients' choice of medical care, we recommend that TCM practitioners and physicians should carefully monitor patient blood glucose levels and the potential side effects of CHP when they are being used alongside or in lieu of diabetes drugs. Further studies are warranted to assess the formulae generally used by TCM doctors in this study in order to determine whether they are really useful as add-on treatments for patients receiving antidiabetic treatment.

The present study has three limitations. First, this study did not include Chinese herbal remedies or decoctions that were purchased directly from TCM herbal pharmacies, nor did we include health foods containing herbs. Thus, the frequency of CHP utilization might have been underestimated. However, because the NHI system covers TCM prescriptions, which generally cost less than the herbs sold in Taiwan's markets, the likelihood that subjects purchased a lot of other herbs outside the NHI database is not high. Second, we are unable to draw any conclusion about the relationship of blood glucose and haemoglobin A1C levels with respect to TCM utilization owing to the lack of actual clinical data. Third, this was a retrospective study and thus does not include a 
randomized placebo group. Thus, great caution is necessary when interpreting the results of the most commonly prescribed Chinese formulae obtained in the present study due to the possibility of a placebo effect.

\section{Conclusions}

Our results suggest that, based on the coexistence of both conventional and traditional Chinese medical treatments, of most the diabetes patients consume herbal therapies with the intention of relieving their diabetes-related symptoms, rather than because they have rejected standard diabetes treatments. Liu-Wei-Di-Huang-Wan and its derivatives are the most frequently prescribed formulae by TCM doctors in Taiwan for diabetes patients. Having recognized the use of TCM, exploring any potential interactions and adverse effects, and integrating both technologies into a holistic treatment system may be beneficial to the overall health, presence of comorbidities, and quality of life, of patients with type 2 diabetes. It is worth noting that although some evidence does support the use of TCM to treat diabetes, the results from the current study may have been confounded by the placebo effect. This emphasizes the need for well-conducted, doubleblind, randomized, placebo-control studies to further evaluate efficacy when Liu-Wei-Di-Huang-Wan is given to patients with type 2 diabetes.

\section{Acknowledgments}

This research was conducted at the Institute of Traditional Medicine at the School of Medicine, National Yang-Ming University, Taipei. The authors would like to express sincere gratitude for the partial support provided for this project in the form of Grants from the Department of Health, Taipei City Government (38), the Committee on Chinese Medicine and Pharmacy (CCMP100-RD-033), and the National Science Council (NSC 99-2320-B-010-011-MY2), Taiwan.

\section{References}

[1] S. Wild, G. Roglic, A. Green, R. Sicree, and H. King, "Global prevalence of diabetes. Estimates for the year 2000 and projections for 2030," Diabetes Care, vol. 27, no. 5, pp. 1047-1053, 2004.

[2] American Diabetes Association, "Economic consequences of diabetes mellitus in the US in 2002," Diabetes Care, vol. 26, no. 3, pp. 917-932, 2003.

[3] C. H. Chang, W. Y. Shau, Y. D. Jiang et al., "Type 2 diabetes prevalence and incidence among adults in Taiwan during 19992004: a national health insurance data set study," Diabetic Medicine, vol. 27, no. 6, pp. 636-643, 2010.

[4] M. I. Harris, R. Klein, T. A. Welborn, and M. W. Knuiman, "Onset of NIDDM occurs at least 4-7 yr before clinical diagnosis," Diabetes Care, vol. 15, no. 7, pp. 815-819, 1992.

[5] W. C. Yang, S. J. Hwang, S. S. Chiang, H. F. Chen, and S. T. Tsai, "The impact of diabetes on economic costs in dialysis patients: experiences in Taiwan," Diabetes Research and Clinical Practice, vol. 54, supplement 1, pp. S47-S54, 2001.
[6] G. Roglic and N. Unwin, "Mortality attributable to diabetes: estimates for the year 2010," Diabetes Research and Clinical Practice, vol. 87, no. 1, pp. 15-19, 2010.

[7] T. H. Lu, C. F. Kwok, and L. T. Ho, "Whether to report diabetes as the underlying cause-of-death? A survey of internists of different sub-specialties," BMC Endocrine Disorders, vol. 10, article 13, 2010.

[8] A. D. Rao, N. Kuhadiya, K. Reynolds, and V. A. Fonseca, "Is the combination of sulfonylureas and metformin associated with an increased risk of cardiovascular disease or all- cause mortality?" Diabetes Care, vol. 31, no. 8, pp. 1672-1678, 2008.

[9] L. L. Lipscombe, "Thiazolidinediones: do harms outweigh benefits?" Canadian Medical Association Journal, vol. 180, no. 1, pp. 16-17, 2009.

[10] Diabetes magazine American Diabetes Association, "Diabetes 101: Metformin,” April 2013, http://forecast.diabetes.org/diabe tes-101/metformin.

[11] W. T. Friedewald, J. B. Buse, J. T. Bigger et al., "Effects of intensive glucose lowering in type 2 diabetes," New England Journal of Medicine, vol. 358, no. 24, pp. 2545-2559, 2008.

[12] A. Patel, S. MacMahon, J. Chalmers et al., "Intensive blood glucose control and vascular outcomes in patients with type 2 diabetes," The New England Journal of Medicine, vol. 358, no. 24, pp. 2560-2572, 2008.

[13] E. S. Huang, S. E. S. Brown, B. G. Ewigman, E. C. Foley, and D. O. Meltzer, "Patient perceptions of quality of life with diabetesrelated complications and treatments," Diabetes Care, vol. 30, no. 10, pp. 2478-2483, 2007.

[14] K. Manya, B. Champion, and T. Dunning, "The use of complementary and alternative medicine among people living with diabetes in Sydney," BMC Complementary and Alternative Medicine, vol. 12, article 2, 2012.

[15] G. Y. Yeh, D. M. Eisenberg, R. B. Davis, and R. S. Phillips, "Use of complementary and alternative medicine among persons with diabetes mellitus: results of a national survey," American Journal of Public Health, vol. 92, no. 10, pp. 1648-1652, 2002.

[16] H. Hikino, M. Yoshizawa, Y. Suzuki, Y. Oshima, and C. Konno, "Isolation and hypoglycemic activity of trichosans A, B, C, D, and E: glycans of Trichosanthes kirilowii roots," Planta Medica, vol. 55, no. 4, pp. 349-350, 1989.

[17] F. L. Xiong, X. H. Sun, L. Gan, X. L. Yang, and H. B. Xu, "Puerarin protects rat pancreatic islets from damage by hydrogen peroxide," European Journal of Pharmacology, vol. 529, no. 1-3, pp. 1-7, 2006.

[18] M. Kako, T. Miura, M. Usami, A. Kato, and S. Kadowaki, "Hypoglycemic effect of the rhizomes of ophiopogonis tuber in normal and diabetic mice," Biological and Pharmaceutical Bulletin, vol. 18, no. 5, pp. 785-787, 1995.

[19] R. Akilen, A. Tsiami, D. Devendra, and N. Robinson, "Glycated haemoglobin and blood pressure-lowering effect of cinnamon in multi-ethnic Type 2 diabetic patients in the UK: a randomized, placebo-controlled, double-blind clinical trial," Diabetic Medicine, vol. 27, no. 10, pp. 1159-1167, 2010.

[20] H. Ping, G. Zhang, and G. Ren, "Antidiabetic effects of cinnamon oil in diabetic KK-A(y) mice," Food and Chemical Toxicology, vol. 48, no. 8, pp. 2344-2349, 2010.

[21] H. J. Kim, M. K. Kong, and Y. C. Kim, "Beneficial effects of Phellodendri Cortex extract on hyperglycemia and diabetic nephropathy in streptozotocin-induced diabetic rats," Journal of Biochemistry and Molecular Biology, vol. 41, no. 10, pp. 710-715, 2008. 
[22] S. S. Liou, I. M. Liu, and M. C. Lai, "The plasma glucose lowering action of Hei-Shug-Pian, the fire-processed product of the root of Aconitum (Aconitum carmichaeli), in streptozotocin-induced diabetic rats," Journal of Ethnopharmacology, vol. 106, no. 2, pp. 256-262, 2006.

[23] R. Zhang, J. Zhou, Z. Jia, Y. Zhang, and G. Gu, "Hypoglycemic effect of Rehmannia glutinosa oligosaccharide in hyperglycemic and alloxan-induced diabetic rats and its mechanism," Journal of Ethnopharmacology, vol. 90, no. 1, pp. 39-43, 2004.

[24] C. C. Chen, C. Y. Hsu, C. Y. Chen, and H. K. Liu, "Fructus Corni suppresses hepatic gluconeogenesis related gene transcription, enhances glucose responsiveness of pancreatic beta-cells, and prevents toxin induced beta-cell death," Journal of Ethnopharmacology, vol. 117, no. 3, pp. 483-490, 2008.

[25] S. H. Chung, C. G. Choi, and S. H. Park, "Comparisons between white ginseng radix and rootlet for antidiabetic activity and mechanism in KKAy mice," Archives of Pharmacal Research, vol. 24, no. 3, pp. 214-218, 2001.

[26] B. S. Ko, J. S. Jang, S. M. Hong et al., "Changes in components, glycyrrhizin and glycyrrhetinic acid, in raw Glycyrrhiza uralensis fisch, modify insulin sensitizing and insulinotropic actions," Bioscience, Biotechnology and Biochemistry, vol. 71, no. 6, pp. 1452-1461, 2007.

[27] J. H. Kim, H. S. Chung, M. Kang et al., "Anti-diabetic effect of standardized herbal formula PM021 consisting of Mori Folium and Aurantii Fructus on type II diabetic Otsuka Long-Evans Tokushima Fatty (OLETF) rats," Diabetes Research and Clinical Practice, vol. 93, no. 2, pp. 198-204, 2011.

[28] O. Potterat, "Goji (Lycium barbarum and L. chinense): phytochemistry, pharmacology and safety in the perspective of traditional uses and recent popularity," Planta Medica, vol. 76, no. 1, pp. 7-19, 2010.

[29] G. S. Birdee and G. Yeh, "Complementary and alternative medicine therapies for diabetes: a Clinical Review," Clinical Diabetes, vol. 28, no. 4, pp. 147-155, 2010.

[30] S. C. Hsieh, J. N. Lai, C. F. Lee, F. C. Hu, W. L. Tseng, and J. D. Wang, "The prescribing of Chinese herbal products in Taiwan: a cross-sectional analysis of the national health insurance reimbursement database," Pharmacoepidemiology and Drug Safety, vol. 17, no. 6, pp. 609-619, 2008.

[31] F. P. Chen, T. J. Chen, Y. Y. Kung et al., "Use frequency of traditional Chinese medicine in Taiwan," BMC Health Services Research, vol. 7, article 26, 2007.

[32] J. N. Lai, C. T. Wu, and J. D. Wang, "Prescription pattern of Chinese herbal products for breast cancer in Taiwan: a population-based study," Evidence-Based Complementary and Alternative Medicine, vol. 2012, Article ID 891893, 7 pages, 2012.

[33] R. C. Fang, Y. T. Tsai, J. N. Lai, C. H. Yeh, and C. T. Wu, “The traditional Chinese medicine prescription pattern of endometriosis patients in Taiwan: a population-based study," EvidenceBased Complementary and Alternative Medicine, vol. 2012, Article ID 591391, 9 pages, 2012.

[34] Y. H. Yang, P. C. Chen, J. D. Wang, C. H. Lee, and J. N. Lai, "Prescription pattern of traditional Chinese medicine for climacteric women in Taiwan," Climacteric, vol. 12, no. 6, pp. 541$547,2009$.

[35] Y. C. Lee, Y. T. Huang, Y. W. Tsai et al., "The impact of universal National Health Insurance on population health: the experience of Taiwan," BMC Health Services Research, vol. 10, article 225, 2010.

[36] N. H. R. Institutes, "National Health Insurance Research database," March 2011, http://nhird.nhri.org.tw/date_01.html.
[37] C. F. D. A. Prevention, "International Classification of Diseases, Ninth Revision (ICD-9)," March 2011, http://www.cdc.gov/nchs /icd/icd9.htm.

[38] C. C. Lin, M. S. Lai, C. Y. Syu, S. C. Chang, and F. Y. Tseng, "Accuracy of diabetes diagnosis in health insurance claims data in Taiwan," Journal of the Formosan Medical Association, vol. 104, no. 3, pp. 157-163, 2005.

[39] K. Chien, T. Cai, H. Hsu et al., "A prediction model for type 2 diabetes risk among Chinese people," Diabetologia, vol. 52, no. 3, pp. 443-450, 2009.

[40] C. H. Tseng, C. P. Tseng, C. K. Chong et al., "Increasing incidence of diagnosed type 2 diabetes in Taiwan: analysis of data from a national cohort," Diabetologia, vol. 49, no. 8, pp. 17551760, 2006.

[41] S. F. Cheng, H. H. Hsu, H. S. Lee, C. S. Lin, Y. C. Chou, and J. H. Tien, "Rational pharmacotherapy in the diabetic hypertension: analysis-prescribing patterns in a general hospital in Taiwan," Journal of Clinical Pharmacy and Therapeutics, vol. 29, no. 6, pp. 547-558, 2004.

[42] UK Prospective Diabetes Study 6, "Complications in newly diagnosed type 2 diabetic patients and their association with different clinical and biochemical risk factors," Diabetes Research, vol. 13, no. 1, pp. 1-11, 1990.

[43] Diabetes UK, "Diabetes in the UK 2010: key statistics on diabetes," March 2010, http://zh.scribd.com/doc/45658612/Diabetes-in-the-UK-2010.

[44] K. Thomas and P. Coleman, "Use of complementary or alternative medicine in a general population in Great Britain. Results from the National Omnibus survey," Journal of Public Health, vol. 26, no. 2, pp. 152-157, 2004.

[45] S. Colagiuri, R. Colagiuri, S. Na'ati, S. Muimuiheata, Z. Hussain, and T. Palu, "The prevalence of diabetes in the Kingdom of Tonga," Diabetes Care, vol. 25, no. 8, pp. 1378-1383, 2002.

[46] D. W. Dunstan, P. Z. Zimmet, T. A. Welborn et al., “The rising prevalence of diabetes and impaired glucose tolerance: the Australian diabetes, obesity and lifestyle study," Diabetes Care, vol. 25, no. 5, pp. 829-834, 2002.

[47] M. Malik, A. Bakir, B. Abi Saab, G. Roglic, and H. King, "Glucose intolerance and associated factors in the multi-ethnic population of the United Arab Emirates: results of a national survey," Diabetes Research and Clinical Practice, vol. 69, no. 2, pp. 188-195, 2005.

[48] G. Danaei, A. B. Friedman, S. Oza, C. J. L. Murray, and M. Ezzati, "Diabetes prevalence and diagnosis in US states: analysis of health surveys," Population Health Metrics, vol. 7, article 1478, p. 16, 2009.

[49] K. C. Wong and Z. Wang, "Prevalence of type 2 diabetes mellitus of Chinese populations in Mainland China, Hong Kong, and Taiwan," Diabetes Research and Clinical Practice, vol. 73, no. 2, pp. 126-134, 2006.

[50] T. Y. Poon, K. L. Ong, and B. M. Cheung, "Review of the effects of the traditional Chinese medicine Rehmannia Six Formula on diabetes mellitus and its complications," Journal of Diabetes, vol. 3, no. 3, pp. 184-200, 2011.

[51] M. B. Covington, "Traditional Chinese medicine in the treatment of diabetes," Diabetes Spectrum, vol. 143, pp. 154-159, 2001.

[52] Committee on Chinese Medicine and Pharmacy, Department of Health, Executive Yuan, Taiwan, "Six-flavour Rehmannia Pill, Rehmannia Bolus with Six Herbs, Liu Wei Ti Huang Wan, Liu Wei Di Huang Wan," April 2013, http://www.ccmp.gov.tw/en/ information/formula_detail.asp?detailno $=19 \&$ selno $=0 \&$ relno $=$ $52 \&$ PageNo $=1$ 
[53] S. Li, B. Zhang, D. Jiang, Y. Wei, and N. Zhang, "Herb network construction and co-module analysis for uncovering the combination rule of traditional Chinese herbal formulae," $B M C$ Bioinformatics, vol. 11, supplement 11, article S6, 2010.

[54] J. J. Shen, C. J. Lin, J. L. Huang, K. H. Hsieh, and M. L. Kuo, “The effect of Liu-Wei-Di-Huang Wan on cytokine gene expression from human peripheral blood lymphocytes," American Journal of Chinese Medicine, vol. 31, no. 2, pp. 247-257, 2003. 


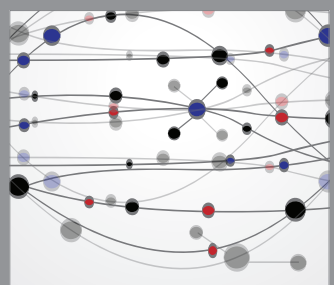

The Scientific World Journal
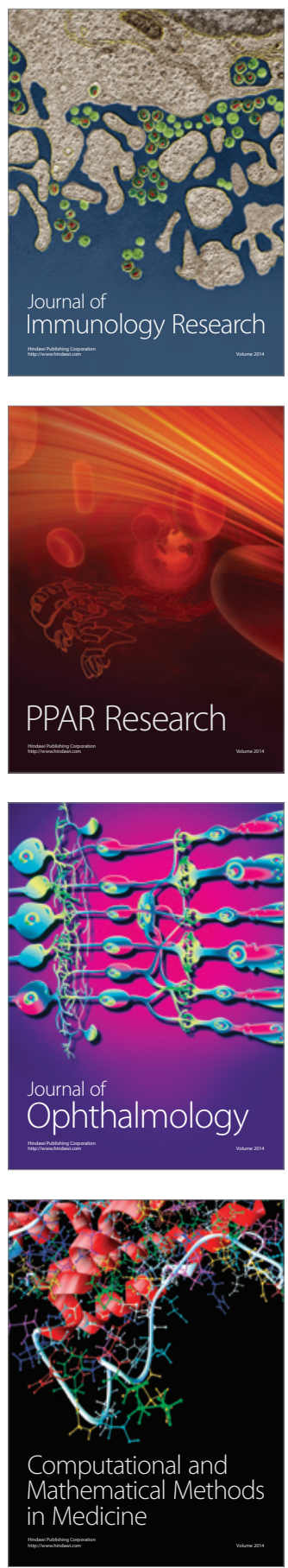

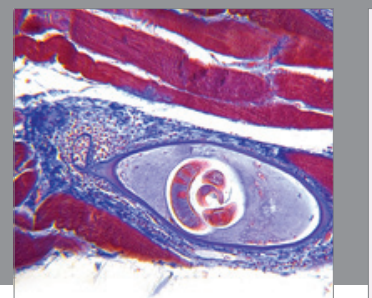

Gastroenterology

Research and Practice
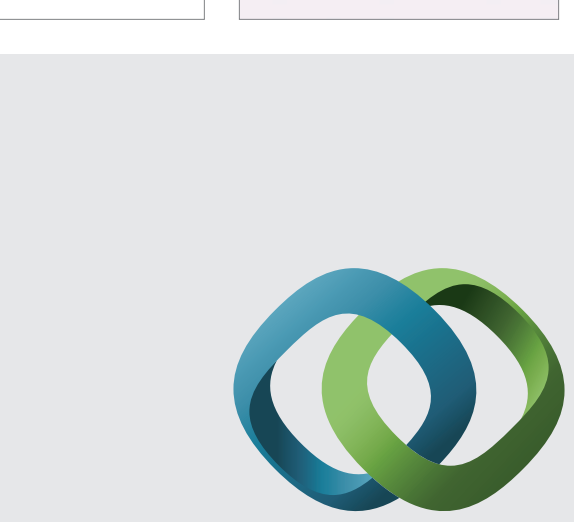

\section{Hindawi}

Submit your manuscripts at

http://www.hindawi.com
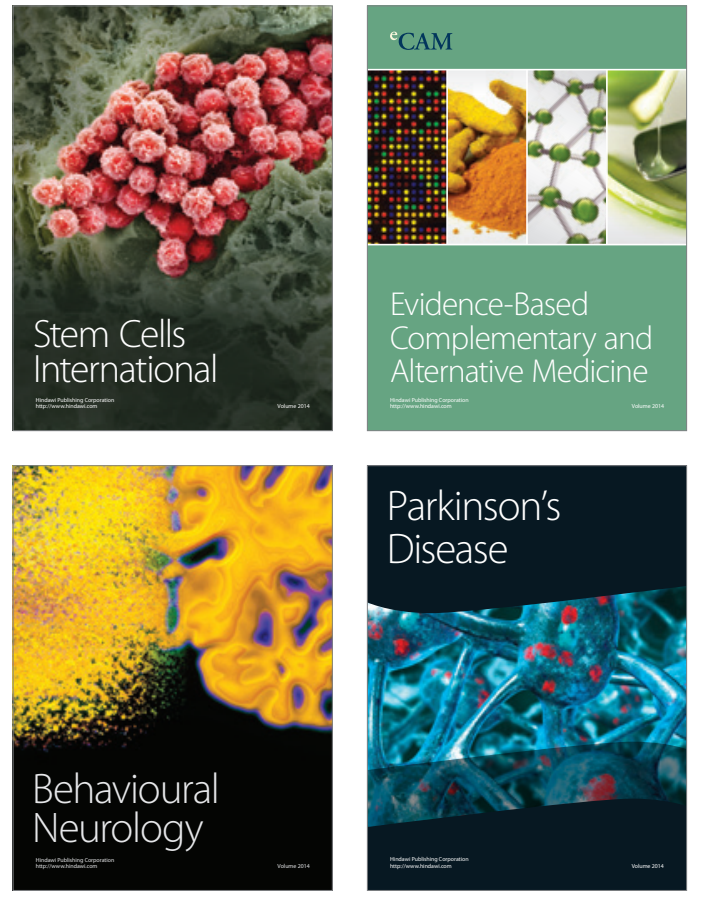
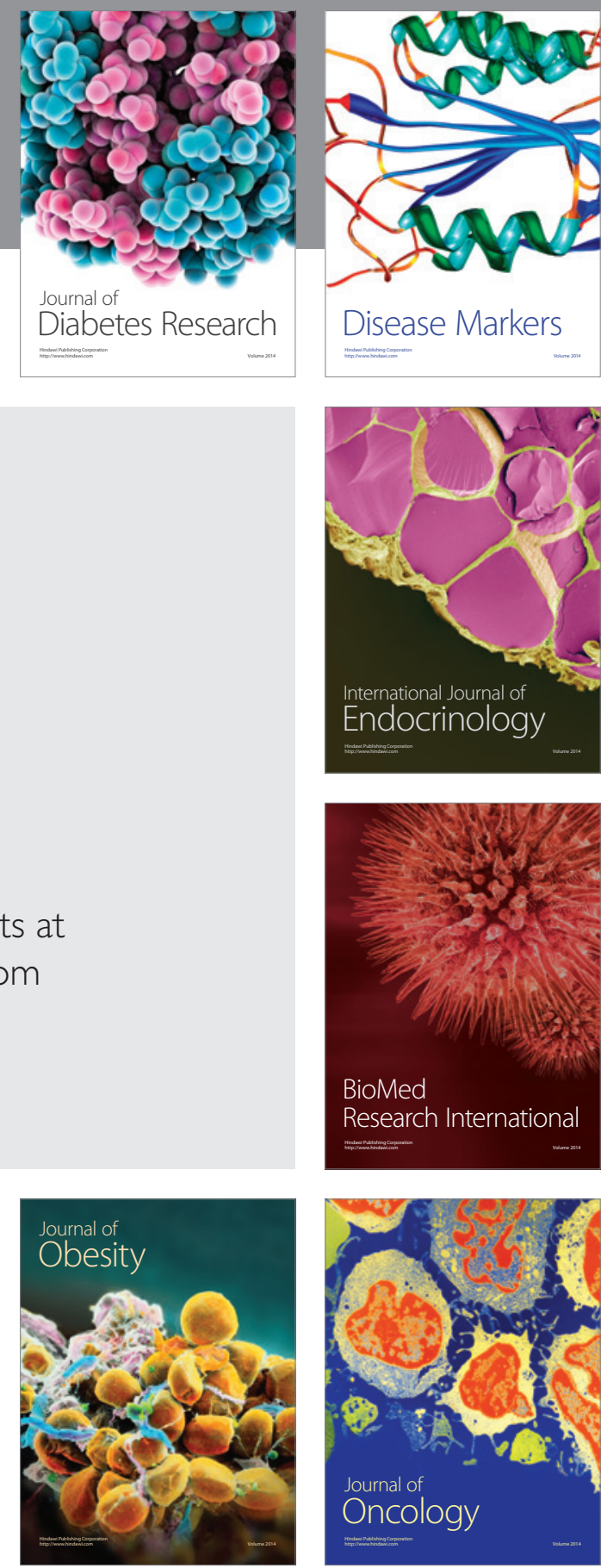

Disease Markers
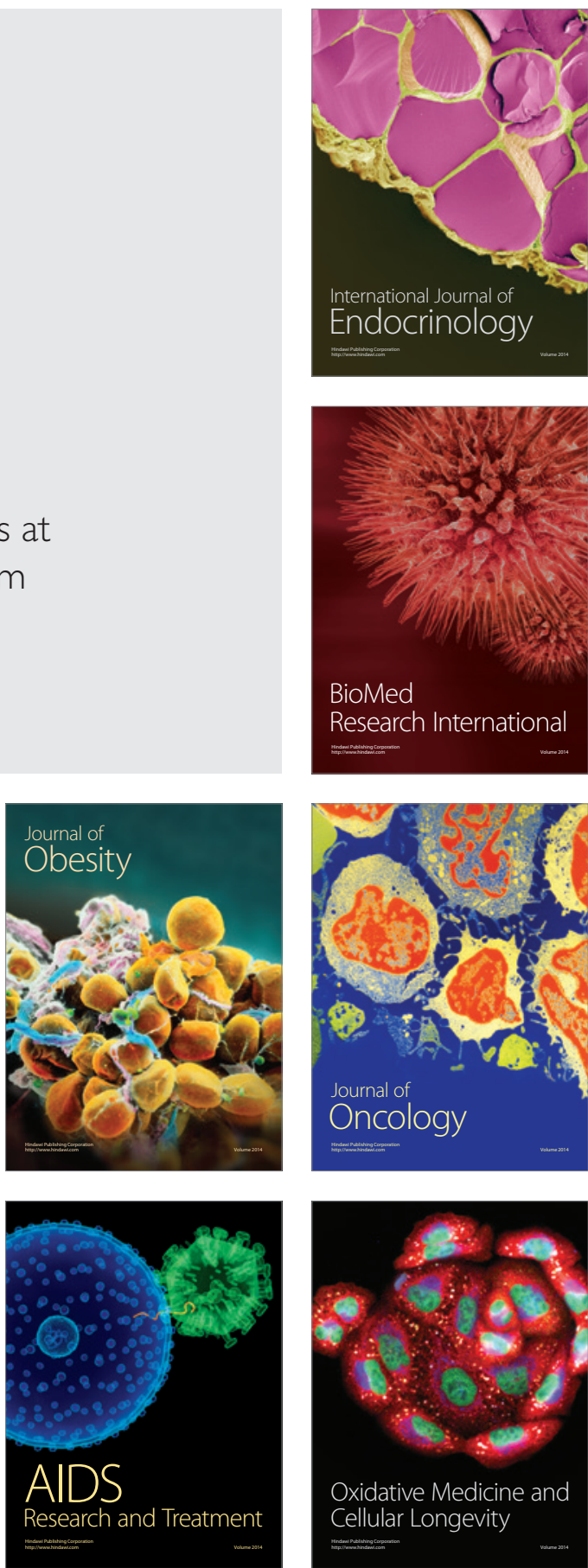